\title{
Synovial plicae of the knee joint: the role of advanced MRI
}

\author{
Katerina Vassiou, ${ }^{1}$ Marianna Vlychou, ${ }^{2}$ Aristidis Zibis, ${ }^{1}$ Athina Nikolopoulou, \\ Ioannis Fezoulidis, ${ }^{2}$ Dimitrios Arvanitis ${ }^{1}$
}

${ }^{1}$ Department of Anatomy, Faculty of Medicine, University of Thessaly, Larissa, Greece ${ }^{2}$ Department of Radiology, University of Thessaly, Larissa, Greece

\section{Correspondence to} Dr Katerina Vassiou, Department of Anatomy, Medical School, University of Thessaly, Biopolis, Larissa 41110, Greece; avassiou@gmail.com

Received 20 June 2013 Revised 12 April 2014 Accepted 4 November 2014 Published Online First 4 December 2014
CrossMark

To cite: Vassiou $\mathrm{K}$, Vlychou M, Zibis A, et al. Postgrad Med J

2015;91:35-40.

\section{ABSTRACT}

Synovial plicae are normal anatomical structures of the knee that may become symptomatic. MRI is an established technique for evaluating the anatomy of the knee, and it is a valuable tool for detecting plicae because of its high resolution resulting in increased tissue characterisation. At MRI, knee plicae appear as low-signal-intensity structures of variable size and thickness, and they are better visualised at fluidsensitive sequences with or without fat suppression. The combined use of clinical examination and MRI may also facilitate the diagnosis of fibrotic or inflamed plicae that may be symptomatic. Arthroscopy remains the gold standard for recognition and repair of knee plicae in cases of knee dysfunction.

\section{INTRODUCTION}

Synovial plicae are anatomically normal structures within the joint capsule of the knee which represent folds of synovial tissue. The most common types according to their anatomical location are suprapatellar, medial, infrapatellar and, much less commonly, lateral. ${ }^{1}$

Controversy exists regarding the clinical importance of a plica, as its presence and size do not necessarily confirm its pathological nature, its correlation with associated symptoms, or the necessity for treatment. ${ }^{1}$ Symptomatic plicae are thickened inflamed structures secondary to knee trauma or other pathological conditions of the knee. ${ }^{2}$ The most commonly symptomatic plica is the medial. ${ }^{3}$ Arthroscopy is considered to be the gold standard for identification of knee plicae and surgical repair when symptomatic. $^{4-6}$ The presence of a symptomatic plica has been correlated with knee impingement syndromes and early osteoarthritis. ${ }^{78}$

Synovial plicae have been described in numerous cadaveric and arthroscopic studies ${ }^{2} 45^{7-15}$ and in some anatomical and clinical studies using MRI, especially MR units of $1.5 \mathrm{~T}^{3}{ }^{10}{ }^{15-18}$ At MRI, plicae are manifested as linear low-signal-intensity structures of varying thickness, and they are better delineated when surrounded by joint fluid. ${ }^{19}$

MRI, most commonly at $1.5 \mathrm{~T}$ and recently at $3 \mathrm{~T}$, has become an established technique for evaluating normal anatomical structures and internal derangements of the knee, and various studies have shown that it is effective in a wide range of clinical settings. MRI and, more specifically, MR arthrography (MRA) are useful tools for detecting plicae because of high resolution and increased tissue characterisation, ${ }^{3} 20$ and aid in the evaluation of pathological synovial plicae and the diagnosis of plica syndrome. ${ }^{17}$ Recognition of normal imaging appearance and location of plicae is important for physicians evaluating MRI scans of the knee. ${ }^{15} \mathrm{~A}$ recent literature review has acknowledged the importance of morphophysiopathology of synovial plicae of the knee in symptomatic patients. ${ }^{21}$ To our knowledge, only one study to date has assessed the detection of infrapatellar synovial plicae at $3 \mathrm{~T}^{10}$

We retrospectively reviewed 437 knees that had undergone MRI scan at $3 \mathrm{~T}$ in our department from June 2010 until June 2012. Patients' demographics and the MRI method used are presented in table 1 . The prevalence of plicae in this population is presented in table 2 .

The objective of the present study was to review the anatomy and embryology of knee plicae and illustrate their morphological features at $3 \mathrm{~T}$ MRI. In addition, we discuss the morphopathology of knee plicae, their correlation with clinical syndromes, and arthroscopic treatment.

\section{Embryology}

There is a debate about the formation of the knee joint during early fetal life. The most widely accepted theory is that, in the 8th week of fetal life, the knee joint is composed of three compartments divided by synovial septa. These compartments are the medial, lateral and supralateral. The synovial septa are partially resorbed after the 12th week of fetal life leading to the formation of plicae. ${ }^{13} 22$ This theory explains the presence of supra- and infra-patellar plicae (IPPs), but it does not explain the formation of the medial or lateral plicae; the existence of a septum dividing the patellofemoral joint into two cavities at a coronal plane has not been verified. ${ }^{1}$ The study of Ogatha and Uhthoff ${ }^{13}$ does not accept the concept of three separate compartments and suggests that the medial plica is not a remnant of a septum but a remnant of mesenchymal tissue that was larger at the medial side of the patellofemoral region associated with the lateralised location of the patella.

\section{MRI features}

At MRI, synovial plicae present as band-like structures of low signal intensity and variable size and thickness. Normal plicae usually appear thin, whereas symptomatic plicae usually appear thickened with or without synovitis. ${ }^{17}$ However, the thickness and size of a plica at MRI are not necessarily indicative of its clinical significance. ${ }^{17}$

The most valuable sequences for the detection of plicae are fluid-sensitive ones including T2-weighted gradient-echo (T2*GRE) and intermediate-weighted proton density (PD) images with or without fat suppression (FS). ${ }^{17}$ The use of spectral FS has been 
Table 1 Patient demographics and MRI protocol at $3 \mathrm{~T}$

\begin{tabular}{|c|c|}
\hline Patients & 437 (281 men, 156 women) \\
\hline Median age & 40.7 years \\
\hline Indications & $\begin{array}{l}\text { Anterior knee pain } \\
\text { Hydrarthrosis } \\
\text { Queries regarding the integrity of menisci and ligaments }\end{array}$ \\
\hline Arthroscopy & 93 cases $(21.3 \%)$ \\
\hline MRI technique & $\begin{array}{l}3 \text { T MR scanner (GE Healthcare, Signa Hdx) } \\
\text { Quadrature coil } \\
\text { Supine position }\end{array}$ \\
\hline MRI protocol & $\begin{array}{l}\text { PD-FS in axial-coronal-sagittal plane } \\
\mathrm{T} 1-\mathrm{SE} \text { in coronal plane } \\
\mathrm{T} 2^{*} \mathrm{GRE} \text { in sagittal plane }\end{array}$ \\
\hline \multicolumn{2}{|c|}{$\begin{array}{l}\text { 1. PD-FS (proton density-fat suppression) in axial-coronal-sagittal plane (slice } \\
\text { thickness }=2-3 \mathrm{~mm} \text {, repetition time }(\mathrm{TR})=2300 \mathrm{~ms} \text {, echo time }(\mathrm{TE})=31 \mathrm{~ms} \text {, field of } \\
\text { view }(\mathrm{FOV})=18 \times 18 \mathrm{~cm} \text {, matrix size }=320 \times 224 \text { and } 384 \times 224) \text {. } \\
\text { 2. } \mathrm{T} 1-\mathrm{SE} \text { in coronal plane }(\mathrm{slice} \text { thickness }=3 \mathrm{~mm}, \mathrm{TR}=660 \mathrm{~ms}, \mathrm{TE}=8.2 \mathrm{~ms} \text {, } \\
\mathrm{FOV}=18 \times 18 \mathrm{~cm} \text {, matrix size }=320 \times 224) \text {. } \\
3 . \mathrm{T} 2^{*} \mathrm{GRE} \text { (gradient echo) in sagittal plane (slice thickness }=4 \mathrm{~mm}, \mathrm{TR}=680 \mathrm{~ms} \text {, } \\
\mathrm{TE}=11.2 \mathrm{~ms}, \mathrm{FOV}=18 \times 18 \mathrm{~cm} \text {, matrix size }=320 \times 224) \text {. }\end{array}$} \\
\hline
\end{tabular}

reported to enhance the contrast between abnormally thickened or oedematous structures and fluid, as well as adjacent normal tissues. The knee protocol used (table 1) is not adapted to the need to demonstrate the anatomy but reflects the routine imaging algorithm that is necessary for diagnosing pathology. The appearance of plicae in the present cohort of patients is presented in table 3 .

The presence of adequate articular fluid is very helpful in the detection of plicae, as their low signal is better visualised within the high-signal-intensity joint fluid. ${ }^{19}$ Intra-articular effusion could play a critical role in enhancing the demonstration of a knee plica; however, even then, normal and abnormal tissue cannot be reliably differentiated. The improved signal-to-noise ratio in $3 \mathrm{~T}$ MR scanners has resulted in an overall better image quality. ${ }^{23}$ Consequently, any possible finding that may indicate an excessive or aberrant anatomical structure and/or associated pathology can be depicted more confidently, thereby increasing the diagnostic efficacy of the radiologist.

MRA with FS is a very useful technique that can be performed on suspicion of clinically significant plicae, when adequate intra-articular fluid is not present. ${ }^{17}$

Most MR studies of the knee, especially those concerning the detection of plicae, have been performed at $1.5 \mathrm{~T}$ MR units. MRI systems of higher magnetic field strength, such as $3 \mathrm{~T} \mathrm{MR}$ units, produce superior image quality due to better signal-to-noise ratio. Consequently, the increased spatial resolution of 3 T MRI results in more accurate visualisation of anatomical structures and improved diagnostic confidence compared with $1.5 \mathrm{~T}$, which positively affects the sensitivity and grading of cartilage lesions of the knee ${ }^{24} 25$ and depiction of normal and abnormal meniscal roots. ${ }^{26} 27$ The use of 3 T MRI

Table 2 Prevalence of plicae

\begin{tabular}{ll}
\hline Type & Prevalence \\
\hline Suprapatellar & $101 / 152(66.45 \%)$ \\
Medial & $81 / 152(53.28 \%)$ \\
Infrapatellar & $27 / 152(17.76 \%)$ \\
Lateral & $2 / 152(1.32 \%)$ \\
Total & $152(34.8 \%)-$ multiple \\
& plicae in $54 / 152(35.5 \%)$ \\
\hline
\end{tabular}

is expected to result in more detailed images with superior anatomical detail, enhancing the possibility of detecting pathology related to plicae impingement. Furthermore, the $3 \mathrm{~T}$ MRI system is cost-effective in the general radiology department for standard knee imaging compared with $1.5 \mathrm{~T}$ because of reduced scan time and the consequent lower cost of examination. ${ }^{28}$

\section{CLINICAL ANATOMY AND CORRELATION WITH MRI}

According to their location, knee plicae are classified as suprapatellar, mediopatellar, infrapatellar or lateral.

The suprapatellar region is the most common location for knee plicae. Mesenchymal tissue in the suprapatellar, mediopatellar and infrapatellar regions that could be considered plicae was found in $33-50 \%$ of fetal specimens in one study. ${ }^{13}$

Plicae are commonly encountered findings at arthroscopy, and their frequency, size, location, names and clinical importance are extremely variable. ${ }^{179}$ In a study of 200 dissections, plicae were found in $90 \%$ of cadavers. ${ }^{1}$ In our study, the most common plica was the suprapatellar, followed by the mediopatellar and IPP, and the least common was the lateral plica (table 2).

Arthroscopy has been reported to be the gold standard for treatment of pathological plicae and is definitely most effective in differential diagnosis between normal and abnormal plicae. ${ }^{5} 6$ The performance of MRI remains controversial in the literature. Jee $e t \mathrm{al}^{3}$ reported a sensitivity of $95 \%$ and a specificity of $72 \%$ for the diagnosis of plicae with a $1.5 \mathrm{~T}$ MR unit when combined axial and sagittal images were used. Nakanishi et al ${ }^{29}$ found the contribution of preoperative MRI useful in the evaluation of the thickness and extension of medial parapatellar plicae, with 93\% sensitivity and 81\% specificity. On the other hand, Uysal et $a l^{30}$ reported that, in a retrospective evaluation of 23 knees with a symptomatic type D medial plica without other intra-articular pathology that underwent arthroscopy, MRI failed to detect pathology in $87 \%$ (ie, 20 cases).

\section{Suprapatellar plica}

The suprapatellar plica is the most common synovial plica of the knee. It extends between the synovium and either the posterior aspect of the quadriceps tendon or the anterior femoral metaphysis. It inserts above the patella and divides the suprapatellar pouch from the knee. ${ }^{17}$

The suprapatellar plica is observed in various forms during arthroscopy ranging from a complete septum to a septum with a perforation either as a fissure, or as complete loss of the septum. ${ }^{17}$ On the basis of anatomical studies, Zidorn subdivided the suprapatellar plica into four types: I, II, III and IV. ${ }^{12}$ In type I (septum completum), the septum completely separates the suprapatellar bursa from the knee joint. In type II (septum perforatum), there are one or more openings of varying size in the plica allowing communication between the suprapatellar bursa and the knee joint cavity. In type III (septum residuale), there is a remaining, usually medially located, fold. In type IV (septum extinctum), the septum is completely involuted.

A prevalence of $55-89 \%$ at autopsy has been reported. ${ }^{1}{ }^{31}$ In cadaveric studies, a suprapatellar plica was observed in $89 \%$ of knees in the study of Harty and Joyce, ${ }^{31}$ and, in the study of Ogata and Uhthoff, ${ }^{13} 33 \%$ of the knees had a suprapatellar plica. Most arthroscopic studies have reported an incidence of 70-91\%. 69 Arthroscopic studies by Dandy ${ }^{9}$ and Kim and Choe, ${ }^{5}$ with 500 and 400 knees, respectively, classified suprapatellar plicae into 10 categories according to their appearance; they reported an incidence of $90 \%$ and $87 \%$, respectively. On the basis of Sakakibara arthroscopic classification, the complete 
Table 3 Plicae appearance

\begin{tabular}{|c|c|c|c|c|}
\hline Characteristic & Suprapatellar plica & Infrapatellar plica & Medial plica & Lateral plica \\
\hline Sequence & PD-FS, T2* GRE & PD-FS, T2* GRE & PD-FS, T2* GRE & PD-FS, T2*GRE \\
\hline Plane & Sagittal & Sagittal & Axial-sagittal & Axial-coronal \\
\hline Signal intensity & Low & Low & Low & Low \\
\hline Location & Posterior and above the patella & $\begin{array}{l}\text { Anterior and parallel to the anterior cruciate } \\
\text { ligament within the Hoffa pad }\end{array}$ & Medial to the patella & Lateral to the patella \\
\hline Shape & Curvilinear structure, variable thickness & Thin linear structure & Thin oblique structure & Thin oblique structure \\
\hline
\end{tabular}

PD-FS, proton density-fat suppression; T2* GRE, gradient echo.

type of suprapatellar plica was found in $12 \%$, and ones with an opening in $20 \% .^{15}$

The suprapatellar plica is usually asymptomatic; however, its detection by MRI is of clinical significance because it can be a cause of anterior knee pain. It is thought that, in such cases, a suprapatellar plica may be the cause of suprapatellar bursitis or chondromalacia. ${ }^{32}$ In cases of persistent anterior knee pain, arthroscopic treatment may be indicated.

At MRI, a suprapatellar plica is seen as a low-signal-intensity band of variable thickness within the high-signal-intensity joint fluid (figure 1A, B). It is better demonstrated in the sagittal plane in fluid-sensitive sequences including intermediateweighted PD images with FS (PD-FS) or gradient echo images (T2*GRE), which are the most useful sequences for its evaluation. The coronal plane is useful in the classification of plicae by Sakakibara. ${ }^{17}$ The prevalence of a suprapatellar plica in the present study (table 1) and its appearance (table 3 ) is in agreement with published data. ${ }^{5}{ }^{17}$

\section{Infrapatellar plica}

The IPP extends between the intercondylar notch, near the anterior cruciate ligament (ACL), and the inferior pole of the patella in the Hoffa fat pad, ${ }^{11}$ with a course anteriorly and inferiorly and, then, superiorly and anteriorly in the Hoffa's fat. ${ }^{17}$ It is located between the medial and lateral compartments as a synovial fold remnant. ${ }^{11}$

The shape of the IPP varies, and it can be classified into five categories according to its morphology: vertical septum, fenestrated, separated from the ACL, split or bipartite, or none of the above. ${ }^{33}$

The first arthroscopic IPP classification was reported by Kim et al. ${ }^{4}$ Dupont $^{1}$ and Ogata and Uhthoff ${ }^{13}$ reported that IPPs are among the most commonly seen plicae. The incidence of IPP is variable, with arthroscopic reports of $85.5 \%{ }^{4}$ and autopsy reports of $50-65 \% .{ }^{9}{ }^{13}$ An IPP is better visualised at arthroscopy than at MRI. ${ }^{3}$ It is believed that MRI underestimates the prevalence of IPP, ${ }^{3}$ as its depiction on routine knee MRI scans is low, with a rate of $0.4 \% .{ }^{18}$ IPP identification on MRI is difficult because of its location and orientation The IPP has two morphological parts: the intercondylar, which can be visualised 'consistently when an effusion is present', and the part that runs in the Hoffa fat pad, which can rarely been visualised. Depending on its morphology, an IPP may also be mistaken for postoperative or post-traumatic changes, for a loose body within the infrapatellar, or for fat pad focal nodular synovitis. In the study of Lee et $a l^{10}{ }^{10}$ a flexed knee and arthrography were used for better IPP detection. This study showed high prevalence $(78.3 \%)$ of the IPP, similar to an arthroscopic study. ${ }^{4}$

An IPP is usually asymptomatic and of little clinical importance, although it has been suggested to be an uncommon cause of knee pain when it is complicated, such as thickened or fibrotic. ${ }^{17}$ Another potential cause of knee pain is injury to the IPP, especially when there is signal abnormality of the plica with no other pathology of the knee joint. ${ }^{16}$
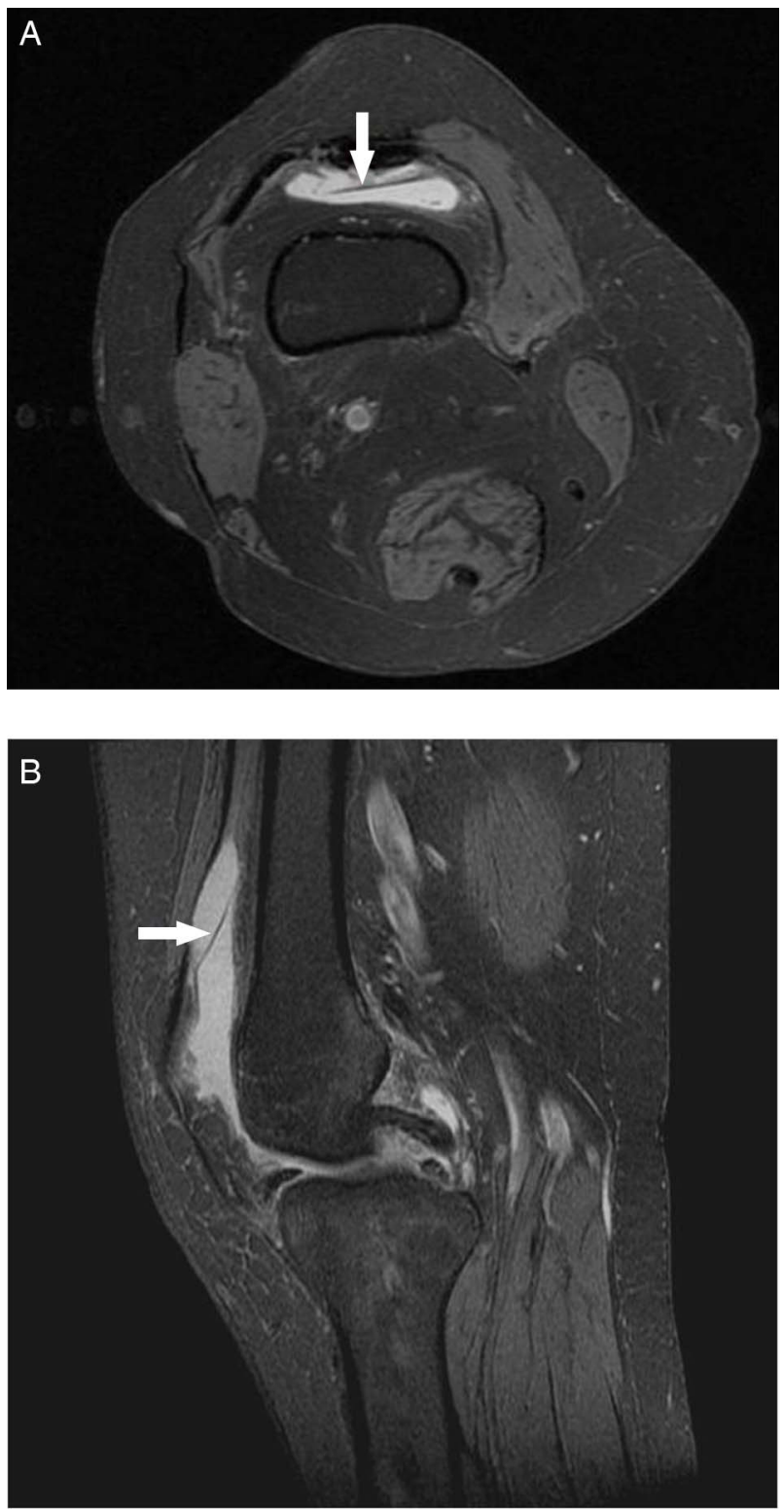

Figure 1 (A) Axial intermediate-weighted MR image with fat suppression shows a suprapatellar plica (arrow) as a band-like stricture of low signal intensity at the suprapatellar pouch, which is filled with fluid. (B) Sagittal intermediate-weighted MR image with fat suppression shows a floating low-signal suprapatellar plica (arrow), which is depicted adequately because of the presence of fluid in the suprapatellar pouch. 

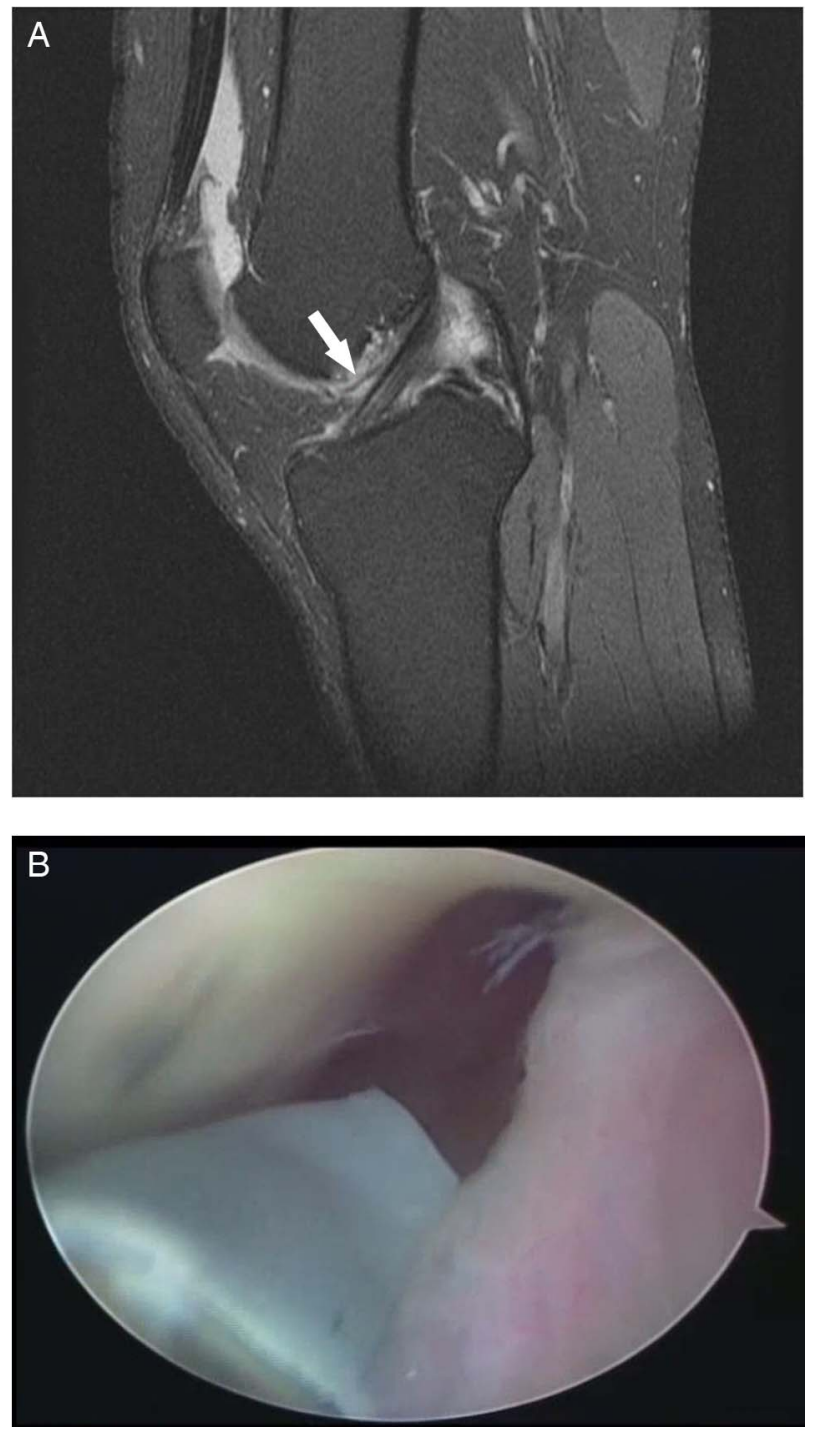

Figure 2 (A) Sagittal MR image with fat suppression shows a linear low-signal-intensity structure anterior and parallel to the anterior cruciate ligament, which represents an infrapatellar plica (arrow). (B) Arthroscopic image of an infrapatellar plica just in front of the anterior cruciate ligament which is not visible.

At MRI, IPP has been detected as a low-signal-intensity band with an anterior and parallel course to the ACL. It is best visualised on both T2* GRE and PD-FS in the sagittal plane ${ }^{17}$ (figure $2 \mathrm{~A}, \mathrm{~B}$ ).

\section{Medial plica}

The medial plica is normally a thin, soft, flexible structure. It originates in the suprapatellar region at the medial aspect of the knee and crosses obliquely inferiorly to the synovium of the infrapatellar fat pad. It is classified into four types on the basis of its arthroscopic appearance: ${ }^{14}$ type A presents with a cordlike elevation in the synovial wall; type B has a shelf-like appearance but does not cover the medial femoral condyle; type $C$ has a shelf-like appearance covering the medial femoral condyle; type D consists of a plica with a central defect. Other classifications of the medial plica have also been reported. In Dandy's classification, the maximum width and the position and incidence of the plica are described. ${ }^{9}$ The most recent and simplified classification is that of Kim and $\mathrm{Choe}^{5}$ in which the medial plica is subdivided into several types according to the extent of its contact with the femoral condyle during flexion.
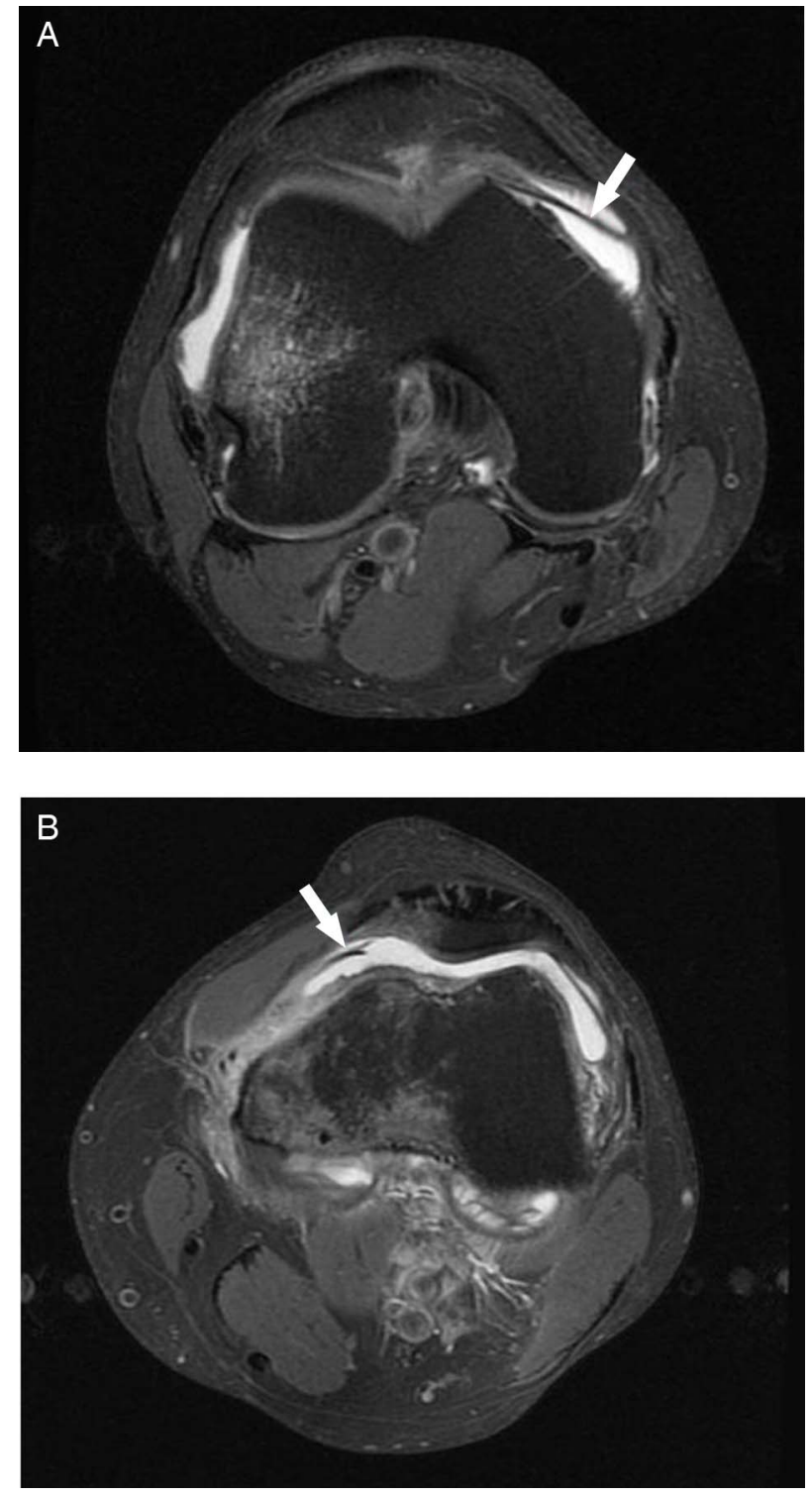

Figure 3 (A) Axial intermediate-weighted MR image with fat suppression reveals a well-demarcated medial plica (arrow) extending toward the midline of the trochlear groove and classified as Sakakibara B. (B) Axial intermediate-weighted MR image with fat suppression indicates a linear structure at the lateral aspect of the suprapatellar pouch that corresponds to a lateral plica (arrow). A medial plica coexists.

The incidence of a medial plica varies between $18 \%$ and $60 \%{ }^{5}$ and $92 \% .^{9}$ The earlier Japanese studies of Sakakibara ${ }^{14}$ reported rates of less than $55 \%$. Furthermore, medial plicae have been found with an incidence of $34 \%$ in cadaveric studies. ${ }^{19} 15$ A medial plica changes orientation and dimensions on knee flexion and extension. ${ }^{34}$

Although the medial plica is not the most common knee plica, it more commonly produces symptoms than the others, especially types $\mathrm{C}$ and $\mathrm{D}$, which may be trapped between the patella and the medial condyle and become thickened. ${ }^{17}$ It may become symptomatic during intense everyday repetitive activities or in association with sports injuries or other causes of trauma, especially if it is thickened. ${ }^{17}$

At MRI, a medial plica is shown as a thin oblique structure of low signal intensity at the medial aspect of the knee joint with variable length depending on Sakakibara classification. ${ }^{17}$ It is 
best defined in the axial and sagittal plane on either T2*GRE or PD-FS sequences (figure 3A).

Medial plica syndrome was defined by Ewing ${ }^{34}$ as 'a painful impairment of knee function in which the only finding to explain the symptoms is the presence of a thickened, hypertrophic plica. ${ }^{35}$ In addition, a medial plica has been implicated in medial compartment osteoarthritis of the knee. ${ }^{36}$ However, not all patients with a medial plica suffer pain or medial patellar syndrome. The symptomatic plica will appear thickened and chronically shows low signal on all MRI sequences because of the formation of fibrous tissue. Plicae are more easily identified in the presence of a joint effusion at MRI. ${ }^{19}$

Diagnosis of medial plica irritation on MRI scans is nonspecific, and the physical examination should be able to demonstrate any significant thickening and fibrosis of a medial synovial plica. MRI scans are more useful in determining if there are other pathologies contributing to medial synovial plica irritation rather than in directly diagnosing pathology in this portion of the knee. ${ }^{37}$ Bone marrow oedema is not directly related to plica syndrome; however, it can be observed in cases of internal derangement or impingement. ${ }^{3} 38$

\section{Lateral plica}

The lateral plica is a longitudinal, very thin structure, located $1-2 \mathrm{~cm}$ lateral to the patella. ${ }^{15}{ }^{17}$ It is considered to be very rare, with an incidence of less than $1 \%{ }^{15}$ Its orientation is in the oblique coronal plane, as it extends between the lateral wall above the popliteal hiatus and its attachment into the synovium at the infrapatellar fat pad. ${ }^{15}$

In the lateral aspect of the knee joint, there may be other synovial structures that should not be confused with a lateral plica. These structures are the lateral alar fold, the superolateral fold and the transverse synovial arcuate fold. Misinterpretation may also apply during MRI analysis. ${ }^{17}$

A lateral plica has been described in an arthroscopic study by Kim and Choe; ${ }^{5}$ it was classified into three types on the basis of its morphology.

At MRI, a lateral plica is seen as an oblique thin low-signal-intensity structure, located lateral to the patella and

\section{Main messages}

- $3 \mathrm{~T} \mathrm{MRI}$ is the standard of reference for assessing the complex anatomy of the knee joint and identifying the presence and location of synovial plicae.

- Imaging of synovial plicae around the knee joint is complementary to clinical examination and may serve as an adjunct prior to any therapeutic interventions, including arthroscopy.

- Anatomical variants of the knee joint including synovial plicae can be assessed by MRI.

\section{Current research questions}

- Is there a role for high-field MR scanners in non-invasive detection of symptomatic plicae around the knee?

- Can imaging replace arthroscopy in the diagnosis of pathological plicae?

- Can imaging serve as a guide for non-surgical therapy of plica syndrome?

\section{Key references}

Sakakibara J. Arthroscopic study on lino's band (plica synovialis mediopatellaris). J Jpn Orthop Assoc 1974;50:513-22.

- Deutsch AL, Resnick D, Dalinka MK, et al. Synovial plicae of the knee. Radiology 1981;141:627-34.

- Garcia-Valtuille R, Abascal F, Cerezal L, et al. Anatomy and MR imaging appearances of synovial plicae of the knee. Radiographics 2002;22:775-84.

- Patel D. Arthroscopy of the plicae-synovial folds and their significance. Am J Sports Med 1978;6:217-25.

- Schindler OS. Synovial plicae of the knee. Curr Orthop 2004;18:210-19.

\section{Self assessment questions}

Please answer true (T) or false (F) to the below,

1. Regarding the synovial plicae around the knee:

A. There are multiple types of plicae and the presence of intra-articular fluid may facilitate their detection

B. The presence of a medial plica is synonymous with plica syndrome

C. Knee plicae should be removed because they cause instability

D. All the above are correct

2. 3 T MRI of the knee joint

A. Is rarely used in routine clinical practice

B. Has the disadvantage of increased scanning time and multiple susceptibility artifacts

C. Is the standard of reference in the assessment of knee joint anatomy

D. None of the above

3. MRI of the knee joint

A. May serve as the only method in the detection and characterisation of symptomatic plicae

B. Is complementary to clinical examination in the diagnosis of plica syndrome

C. Should always be accompanied by MR arthrography

D. Is not well tolerated by the majority of patients

4. Infrapatellar plica

A. Is located at the suprapatellar pouch

B. Is the most common synovial plica

C. Is located at the Hoffa fat pad near the anterior cruciate ligament

D. Is responsible for plica syndrome

5. Medial plica
A. Is classified into four types according to Sakakibara
B. Is the most common symptomatic plica
C. Is considered to be very rare with an incidence of $<1 \%$
D. A and B are correct

best depicted in the axial and coronal plane on either T2*GRE or PD-FS sequences (figure $3 \mathrm{~B}$ ).

\section{CONCLUSION}

Synovial plicae of the knee are normal anatomical structures that are usually asymptomatic but occasionally may be involved 
in knee pathologies. MRI can be used for the identification and characterisation of synovial plicae, and 3 T MRI may improve the detection of these structures because of the higher signal-to-noise ratio and spatial resolution, especially in the presence of increased intra-articular fluid. In a symptomatic clinical setting, MRI can be an adjunct to clinical examination and may provide information that could be useful for pre-surgical planning.

Contributors KV, MV AZ and AN specified the content of the manuscript, identified the relevant articles for inclusion, reviewed all drafts, and revised critically for important intellectual content. IF and DA approved the version submitted.

\section{Competing interests None.}

Ethics approval Ethics committee of the University Hospital of Larissa, Greece.

Provenance and peer review Not commissioned; externally peer reviewed.

\section{REFERENCES}

1 Dupont JY. Synovial plicae of the knee. Controversies and review. Clin Sports Med 1997;16:87-122.

2 Hardaker WT, Whipple TL, Bassett FH III. Diagnosis and treatment of the plica syndrome of the knee. J Bone Joint Surg Am 1980;62:221-5.

3 Jee WH, Choe BY, Kim JM, et al. The plica syndrome: diagnostic value of MRI with arthroscopic correlation. J Comput Assist Tomogr 1998;22:814-18.

$4 \mathrm{Kim} \mathrm{SJ}$, Min BH, Kim HK. Arthroscopic anatomy of the infrapatellar plica. Arthroscopy 1996;12:561-4.

$5 \mathrm{Kim}$ SJ, Choe WS. Arthroscopic findings of the synovial plicae of the knee. Arthroscopy 1997;13:33-41.

6 Patel D. Arthroscopy of the plicae-synovial folds and their significance. Am J Sports Med 1978:6:217-25.

7 Munzinger $\mathrm{U}$, Ruckstuhl J, Scherrer $\mathrm{H}$, et al. Internal derangement of the knee joint due to pathologic synovial folds: the mediopatellar plica syndrome. Clin Orthop Relat Res 1981:59-64.

8 Kent M, Khanduja V. Synovial plicae around the knee. Knee 2010;17:97-102.

9 Dandy DJ. Anatomy of the medial suprapatellar plica and medial synovial shelf. Arthroscopy 1990;6:79-85.

10 Lee $\mathrm{YH}$, Song HT, Kim S, et al. Infrapatellar plica of the knee: revisited with MR arthrographies undertaken in the knee flexion position mimicking operative arthroscopic posture. Eur J Radiol 2012;81:2783-7.

11 Al-Hadithy N, Gikas P, Mahapatra AM, et al. Review article: plica syndrome of the knee. J Orthop Surg (Hong Kong) 2011;19:354-8.

12 Zidorn T. Classification of the suprapatellar septum considering ontogenetic development. Arthroscopy 1992;8:459-64.

13 Ogata S, Uhthoff HK. The development of synovial plicae in human knee joints: an embryologic study. Arthroscopy 1990;6:315-21.

14 Sakakibara J. Arthroscopic study on lino's band (plica synovialis mediopatellaris). I Jpn Orthop Assoc 1974;50:513-22.

15 Boles CA, Martin DF. Synovial plicae in the knee. AJR Am J Roentgenol 2001;177:221-7.

16 Cothran RL, McGuire PM, Helms CA, et al. MR imaging of infrapatellar plica injury. AJR Am J Roentgenol 2003;180:1443-7.

17 Garcia-Valtuille R, Abascal F, Cerezal L, et al. Anatomy and MR imaging appearances of synovial plicae of the knee. Radiographics 2002;22:775-84.

18 Kosarek FJ, Helms CA. The MR appearance of the infrapatellar plica. AJR Am J Roentgenol 1999;172:481-4.
19 Hopper MA, Grainger AJ. Knee injuries. In: Robinson P, ed. Essential radiology for sports medicine. Springer, 2010:1-28.

20 Jemelik P, Strover AE, Evans G. Results of resection of medial patellar plica through a supero-lateral portal as a main arthroscopic procedure. Acta Chir Orthop Traumatol Cech 2008;75:369-74.

21 Silva E, da Silva A, Gomez C. Morphophysiopathology of synovial plica from knee joint: a literature review. J Morphol Sci 2012;29:69-75.

22 Gray DJ, Gardner E. Prenatal development of the human knee and superior tibiofibular joints. Am J Anat 1950;86:235-87.

23 Lavdas E, Vlychou M, Arikidis N, et al. Comparison of T1-weighted fast spin-echo and T1-weighted fluid-attenuated inversion recovery images of the lumbar spine at 3.0 Tesla. Acta Radiol 2010:51:290-5.

24 Kijowski R, Blankenbaker DG, Davis KW, et al. Comparison of 1.5- and 3.0-T MR imaging for evaluating the articular cartilage of the knee joint. Radiology 2009;250:839-48.

25 Wong S, Steinbach L, Zhao J, et al. Comparative study of imaging at 3.0T versus 1.5T of the knee. Skeletal Radiol 2009;38:761-9.

26 You MW, Park J, Park S, et al. Posterior root of lateral meniscus: the detailed anatomic description on 3T MRI. Acta Radiol 2014;55:359-65.

27 Ren AH, Zheng ZZ, Shang $Y$, et al. An anatomical study of normal meniscal roots with isotropic 3D MRI at 3T. Eur J Radiol 2012;81:e783-8.

28 Wiersma HW, Westerbeek RE, de Leeuw LA, et al. Cost-effectiveness of 3T MRI versus $1.5 \mathrm{~T}$ in imaging of the knee in general radiology practice. Fortschr Röntgenstr 2010:182-A11.

29 Nakanishi K, Inoue M, Ishida T, et al. MR evaluation of mediopatellar plica. Acta Radiol 1996;37:567-1.

30 Uysal M, Asik M, Akpinar S, et al. Arthroscopic treatment of symptomatic type D medial plica. Int Orthop 2008;32:799-804.

31 Harty M, Joyce JJ III. Synovial folds in the knee joint. Orthop Rev 1977;7: 91-8.

32 Pekmezci M, Atay $\mathrm{OA}$, Kerimoglu U, et al. A complete supra-patellar plica with an unusual presentation. Knee Surg Sports Traumatol Arthrosc 2006;14:872-4.

33 Demirag B, Ozturk C, Karakayali M. Symptomatic infrapatellar plica. Knee Surg Sports Traumatol Arthrosc 2006;14:156-0.

34 Ewing JW. Plica: pathologic or not? J Am Acad Orthop Surg 1993;1: $117-21$.

35 Lyu SR, Chiang JK, Tseng CE. Medial plica in patients with knee osteoarthritis: a histomorphological study. Knee Surg Sports Traumatol Arthrosc 2009;18: 769-76.

36 Shetty VD, Vowler SL, Krishnamurthy S, et al. Clinical diagnosis of medial plica syndrome of the knee: a prospective study. J Knee Surg 2007;20:277-80.

37 Griffith CJ, LaPrade RF. Medial plica irritation: diagnosis and treatment. Curr Rev Musculoskelet Med 2008;1:53-60.

38 Schindler OS. Synovial plicae of the knee. Curr Orthop 2004;18:210-19.

\section{Answers}

1. (A) $\mathrm{T} ;(\mathrm{B}) \mathrm{F}$; (C) $\mathrm{F}$; (D) $\mathrm{F}$

2. (A) $F ;(B) F ;(C) T ;(D) F$

3. (A) $\mathrm{F} ;(\mathrm{B}) \mathrm{T}$; (C) $\mathrm{F}$; (D) F

4. (A) $\mathrm{F}$; (B) $\mathrm{F}$; (C) $\mathrm{T}$; (D) $\mathrm{F}$

5. (A) $F$; (B) F; (C) F; (D) $T$ 